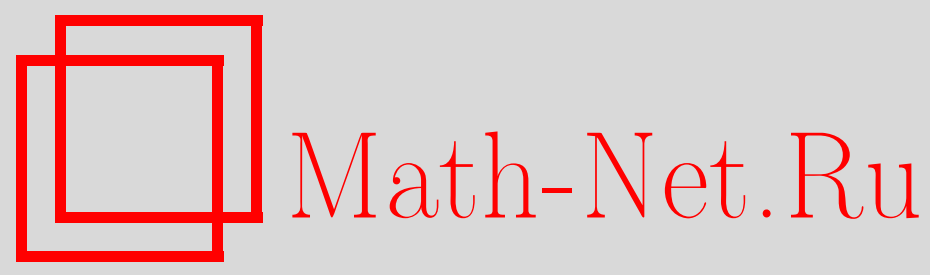

В. В. Гончаров, С. А. Тимошин, Об одном классе эволюционных уравнений: существование решений с функциональными краевыми условиями, Матем. заметки, 1999, том 65, выпуск 1, 48-60

DOI: https://doi.org/10.4213/mzm1027

Использование Общероссийского математического портала Math-Net.Ru подразумевает, что вы прочитали и согласны с пользовательским соглашением http://www.mathnet.ru/rus/agreement

Параметры загрузки:

IP : 3.80 .253 .173

26 апреля 2023 г., 14:04:06

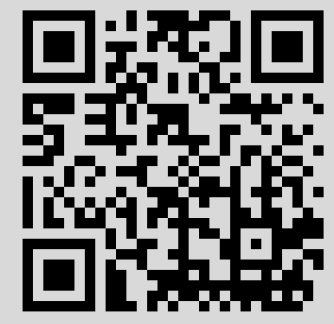




\title{
ОБ ОДНОМ КЛАССЕ ЭВОЛЮЦИОННЫХ УРАВНЕНИЙ: СУЩЕСТВОВАНИЕ РЕШЕНИЙ \\ С ФУНКЦИОНАЛЬНЫМИ КРАЕВЫМИ УСЛОВИЯМИ
}

\author{
В.В. Гончаров, С. А. Тимошин
}

\begin{abstract}
Рассматривается эволюционное уравнение, правая часть которого представляет собой сумму линейного неограниченного оператора, порождающего компактную сильно непрерьвную полугруппу, и некоторого непрерьвного оператора, действующего в функциональных пространствах. Доказано существование решения, которое не выходит за пределы фиксированного замкнутого выпуклого множества и удовлетворяет дополнительно функциональному краевому условию, частньми случаями которого являются начальное условие Коши, условие периодичности, смешанное условие, включающее в себя непрерывное преобразование пространственных переменных, и т.д. Основной результат проиллюстрирован на примере краевой задачи для дифференциально-операторного уравнения с частными производными.
\end{abstract}

Библиограф̆ия: 16 названий.

1. Введение. Пусть $T=[0,1]$ - отрезок числовой прямой $\mathbb{R}$ с мерой Лебега $\mu(d t)$ и $X$ - банахово пространство с нормой $\|\cdot\|$. Как обычно, через $L_{1}(T, X)$ будем обозначать пространство классов эквивалентности интегрируемых по Бохнеру функций $u: T \rightarrow X$, снабженное нормой $\|\cdot\|_{1}$,

$$
\|u\|_{1}=\int_{T}\|u(t)\| d t .
$$

В работе рассматривается эволюционное уравнение

$$
\dot{u}(t)=\boldsymbol{A} u(t)+g(u)(t)
$$

где $\boldsymbol{A}$ - линейный (вообще говоря, неограниченный) оператор на $X$, порождающий сильно непрерывную полугрупу, а оператор $g$ переводит некоторое множество $\mathscr{U} \subset L_{1}(T, X)$ в $L_{1}(T, X)$. Это уравнение мы будем называть дифференииально-операторным уравнением, подчеркивая тем самым специфику входящих в него объектов. В частном случае, когда $g$ представляет собой оператор Немьщкого, соответствующий функции $f$ : $T \times X \rightarrow X$, соотношение вида (1) часто называют полулинейным әволющионным

Работа выполнена при финансовой поддержке Российского фонда фундаментальных исследований, грант № 96-01-00326. 
уравнением. Применяя подход, основанньй на непрерьвных селекторах и теореме Шаудера о неподвижной точке (подобно тому, как это делалось в [1], [2]), мы докажем существование интегрального решения $u(\cdot)$ уравнения $(1)$, удовлетворяющего фазовому ограничению

$$
u(t) \in K \quad \text { для всех } t \in T,
$$

где $K$ - фиксированное замкнутое подмножество $X$, и краевому условию

$$
u(0)=\varphi(u)
$$

в котором отображение $\varphi$ определено на подмножестве пространства $L_{1}(T, X)$ и принимает значения в $X$.

Если отображение $\varphi$ постоянно, $\varphi(u) \equiv u_{0} \in X$, то мы приходим к обычной начальной задаче, которая для полулинейных уравнений изучалась многими авторами. Ими рассматривались вопросы существования, единственности и регулярности решения (в том числе, выживающего на замкнутом множестве) при различных предположениях на функцию $f$ и на порождаемую оператором $\boldsymbol{A}$ полугруппу. Отметим работы [3]-[5], содержащие принципиальные результаты в этом направлении, многочисленные приложения к краевым задачам для дифференциальных уравнений с частными производными, а также обширную библиографию.

Другой распространенньй пример функциональных условий (3) дает условие периодичности $u(0)=u(\theta), 0<\theta \leqslant 1$. Для полулинейных эволюционных уравнений в [6], [7] доказаны теоремы сушествования периодических решений, гарантирующие, кроме того, попадание решения в фиксированное замкнутое множество $K$. В автономном случае из этих теорем непосредственно следует сушествование точек равновесия.

Среди работ, посвященных вопросам разрешимости функциональных краевых задач общего вида, отметим серию статей [8]-[10] С. А. Брыкалова, в которых наряду с традиционными двухточечньми краевьми условиями рассматриваются интегральные условия, условия типа функциональных норм и т.д. При этом, однако, правая часть дифференциально-операторного уравнения предполагается непрерьвной.

Поскольку оператор $g$ в (1) зависит, вообще говоря, не только от значения $u(\cdot)$ в момент времени $t$, а от всех значений функции, то полученные в настоящей статье результаты могут быть использованы как для исследования различных интегро-дифференциальных уравнений (в том числе, с частньми производными), так и для доказательства существования решений широкого класса эволюционных включений с невьпуклозначной правой частью.

2. Некоторые свойства полугрупп линейных ограниченных операторов. Напомним, что однопараметрическое семейство $S=\{S(t), t \geqslant 0\}$ линейных ограниченных операторов на $X$ называется сильно непрерывной полугруппой ( $c_{0}$-полугруппой), если

1) $S(t+\tau)=S(t) S(\tau)$ для всех $t, \tau \geqslant 0$;

2) $S(0)=J$ - тождественньй оператор на $X$;

3) $\lim _{t \rightarrow 0+} S(t) x=x$ для каждого $x \in X$.

Основные сведения из теории полугрупп линейных операторов можно найти, например, в книге [3]. Известно, в частности, что для любой $c_{0}$-полугруппы $S$ существуют константы $M \geqslant 1$ и $\omega \geqslant 0$ такие, что

$$
\|S(t)\| \leqslant M e^{\omega t} \quad \text { для всех } t \geqslant 0,
$$


где $\|$ • || означает также норму оператора. Полугруппа назьвается сжиммающей, если $M=1, \omega=0$. С каждой сильно непрерьвной полугруппой $S$ можно связать линейньй (вообще говоря, неограниченньй) оператор $\boldsymbol{A}: D(\boldsymbol{A}) \rightarrow X$, определенный на множестве

$$
D(\boldsymbol{A}):=\left\{x \in X: \text { существует предел } \lim _{t \rightarrow 0+} \frac{S(t) x-x}{t}\right\},
$$

плотном в $X$, по правилу

$$
\boldsymbol{A} x:=\lim _{t \rightarrow 0+} \frac{S(t) x-x}{t}, \quad x \in D(\boldsymbol{A}) .
$$

Принято говорить, что $\boldsymbol{A}$ - инфинитезимальный генератор полугрупшы $S=\{S(t)$, $t \geqslant 0\}$.

Полугруппа $S$ называется дифферениируемой, если $S(t) x \in D(\boldsymbol{A})$ для всех $x \in X$, $t>0 ;$ аналитической, если она дифференцируема и существует $N>0$ такое, что $\|\boldsymbol{A} S(t)\| \leqslant N / t$ для всех $t \in(0,1] ;$ компактной, если оператор $S(t): X \rightarrow X$ является компактным при каждом $t>0$.

Наряду с банаховым пространством $L_{1}(T, X)$ в дальнейшем мы будем использовать пространство $C(T, X)$ непрерьвных функций $u: T \rightarrow X$ с топологией равномерной сходимости на компактах из полуинтервала $(0,1]$. Последняя топология (обозначим ее через $\mathscr{T}_{c}$ ) может быть задана с помошью счетного семейства полунорм

$$
\left\{p_{m}(\cdot), m=1,2, \ldots\right\}, \quad p_{m}(u):=\sup _{t \in[1 / m, 1]}\|u(t)\|
$$

а потому метризуема. Тот факт, что множество $\mathcal{U} \subset C(T, X)$ рассматривается как самостоятельное пространство относительно топологии, индуцированной $\mathscr{T}_{c}$ (нормой пространства $\left.L_{1}(T, X)\right)$, мы будем отражать в записи $\left(\mathscr{U}, \mathscr{T}_{c}\right)\left(\right.$ соответственно $\left.\left(\mathscr{U},\|\cdot\|_{1}\right)\right)$.

Множество $Q \subset L_{1}(T, X)$ назовем равномерно интегрируемым, если для любого $\varepsilon>0$ существует $\delta>0$ такое, что

$$
\int_{E}\|v(t)\| d t \leqslant \varepsilon
$$

как только $v(\cdot) \in Q$ и $E \subset T$ - измеримое множество с $\mu(E) \leqslant \delta$.

Для фиксированной полугрупшы $S$ определим оператор $\mathscr{P}_{S}: X \times L_{1}(T, X) \rightarrow L_{1}(T, X)$ :

$$
\mathscr{P}_{S}(\xi, v(\cdot))(t):=S(t) \xi+\int_{0}^{t} S(t-\tau) v(\tau) d \tau, \quad \xi \in X, \quad v(\cdot) \in L_{1}(T, X), \quad t \in T
$$

Установим теперь одно свойство компактности, которое будет играть важную роль в дальнейших построениях. Под мерой некомпактности ограниченного множества $C \subset X$ понимается число $\varkappa(C)$, равное точной нижней грани всех $r>0$ таких, что $C$ может быть покрыто конечным числом шаров, радиус которых не превосходит $r$. Свойства меры некомпактности анонсированы, например, в [11]. 
Лемма 1. Пусть $S$ - компактная $c_{0}$-полугруппа линейных ограниченных операторов на $X$. Тогда для любого ограниченного $K \subset X$ и любого равномерно интегрируемого $Q \subset L_{1}(T, X)$ оператор $\mathscr{P}_{S}$ переводит множсество $K \times Q$ в относительно компактное подмножество пространства $\left(C(T, X), \mathscr{T}_{c}\right)$.

ДокАЗАТЕЛьство. Из (4) непосредственно следует

$$
\left\|\mathscr{P}_{S}(\xi, v(\cdot))(t)\right\| \leqslant M e^{\omega t}\left(\|\xi\|+\int_{T}\|v(\tau)\| d \tau\right)
$$

Так как равномерно интегрируемое множество, очевидно, ограничено в $L_{1}(T, X)$, то $\Sigma:=\left\{\mathscr{P}_{S}(\xi, v(\cdot))(t): \xi \in K, v(\cdot) \in Q, t \in T\right\}$ ограничено в $X$. Зафиксируем $t \in T$, $t>0$, и покажем относительную компактность в $X$ множества $\mathscr{P}_{S}(K \times Q)(t) \subset \Sigma$. Выбрав достаточно малое $h>0, t-h>0$, и воспользовавшись свойствами функции $\varkappa(\cdot)$, получим

$\varkappa\left(\mathscr{P}_{S}(K \times Q)(t)\right) \leqslant \varkappa\left(S(h) \mathscr{P}_{S}(K \times Q)(t-h)\right)+\varkappa\left(\left\{\int_{t-h}^{t} S(t-\tau) v(\tau) d \tau: v(\cdot) \in Q\right\}\right)$.

Первое слагаемое в правой части равно нулю в силу компактности оператора $S(h)$ и ограниченности множества $\mathscr{P}_{S}(K \times Q)(t-h) \subset \Sigma$. Оценим второе слагаемое [11, с. 19]:

$$
\varkappa\left(\left\{\int_{t-h}^{t} S(t-\tau) v(\tau) d \tau: v(\cdot) \in Q\right\}\right) \leqslant 2 M e^{\omega} \sup _{v(\cdot) \in Q} \int_{t-h}^{t}\|v(\tau)\| d \tau .
$$

Согласно равномерной интегрируемости $Q$ для любого $\varepsilon>0$ найдется $h_{0}>0$ такое, что

$$
\varkappa\left(\left\{\int_{t-h}^{t} S(t-\tau) v(\tau) d \tau: v(\cdot) \in Q\right\}\right) \leqslant \varepsilon
$$

при всех $0<h \leqslant h_{0}$. Поскольку левая часть неравенства (5) не зависит от $h$, то $\varkappa\left(\mathscr{P}_{S}(K \times Q)(t)\right)=0$ и множество относительно компактно.

Возьмем $t_{0} \in(0,1]$ и докажем равностепенную непрерьвность семейства функций $\mathscr{P}_{S}(K \times Q)$ в этой точке справа. Зафиксировав $\varepsilon>0$, по доказанному вьше найдем конечное число точек $x_{i}, i=1, \ldots, n$, таких, что

$$
\mathscr{P}_{S}(K \times Q)\left(t_{0}\right) \subset \bigcup_{i=1}^{n}\left(x_{i}+\frac{\varepsilon}{3\left(M e^{\omega}+1\right)} \bar{B}\right) .
$$

Через $\bar{B}$ здесь и далее мы обозначаем замкнутьй единичный шар с центром в 0 из $X$. В силу свойств полугрупшы $S$ и множества $Q$ существует $\delta>0$, для которого $\| S\left(t-t_{0}\right) x_{i}-$ $x_{i} \| \leqslant \varepsilon / 3, i=1, \ldots, n$, и

$$
\int_{t_{0}}^{t}\|v(\tau)\| d \tau \leqslant \frac{\varepsilon}{3 M e^{\omega}}
$$

при всех $v(\cdot) \in Q$ и $t \in T, t_{0} \leqslant t \leqslant t_{0}+\delta$. Тогда для $\xi \in K$ и $v(\cdot) \in Q$ выберем точку $x_{i}$,

$$
\left\|\mathscr{P}_{S}(\xi, v(\cdot))\left(t_{0}\right)-x_{i}\right\| \leqslant \frac{\varepsilon}{3\left(M e^{\omega}+1\right)}
$$


и для любого $t \in T, t_{0} \leqslant t \leqslant t_{0}+\delta$, будем иметь

$$
\begin{aligned}
&\left\|\mathscr{P}_{S}(\xi, v(\cdot))(t)-\mathscr{P}_{S}(\xi, v(\cdot))\left(t_{0}\right)\right\| \\
& \leqslant\left\|S\left(t_{0}\right)\left(S\left(t-t_{0}\right) \xi-\xi\right)+\int_{0}^{t_{0}} S\left(t_{0}-\tau\right)\left(S\left(t-t_{0}\right) v(\tau)-v(\tau)\right) d \tau\right\| \\
&+\int_{t_{0}}^{t}\|S(t-\tau) v(\tau)\| d \tau \\
& \leqslant\left\|S\left(t-t_{0}\right)-J\right\|\left\|\mathscr{P}_{S}(\xi, v(\cdot))\left(t_{0}\right)-x_{i}\right\| \\
&+\left\|S\left(t-t_{0}\right) x_{i}-x_{i}\right\|+M e^{\omega} \int_{t_{0}}^{t}\|v(\tau)\| d \tau \leqslant \varepsilon .
\end{aligned}
$$

Для того чтобы доказать равностепенную непрерьвность $\mathscr{P}_{S}(K \times Q)$ слева, возьмем $h>0, t_{0}-2 h \geqslant 0$, настолько малое, что

$$
\int_{t-h}^{t}\|v(\tau)\| d \tau \leqslant \frac{\varepsilon}{8 M e^{\omega}}
$$

для всех $v(\cdot) \in Q$ и $t \in T, t-h>0$. Аналогично предыдущему найдутся точки $x_{i} \in X$, $i=1, \ldots, n$, и число $0<\delta \leqslant h$ такие, что

$$
\begin{gathered}
S(h) \Sigma \subset \bigcup_{i=1}^{n}\left(x_{i}+\frac{\varepsilon}{8\left(M e^{\omega}+1\right)} \bar{B}\right), \quad\left\|S\left(t_{0}-t\right) x_{i}-x_{i}\right\| \leqslant \frac{\varepsilon}{4}, \quad i=1, \ldots, n, \\
\int_{t}^{t_{0}}\|v(\tau)\| d \tau \leqslant \frac{\varepsilon}{4 M e^{\omega}}
\end{gathered}
$$

для всех $v(\cdot) \in Q$ и $t \in T, t_{0}-\delta \leqslant t \leqslant t_{0}$. Пусть теперь $\xi \in K, v(\cdot) \in Q$ и $t \in\left[t_{0}-\delta, t_{0}\right]$. Выбирая $x_{i}$ из условия

$$
\left\|S(h) \mathscr{P}_{S}(\xi, v(\cdot))(t-h)-x_{i}\right\| \leqslant \frac{\varepsilon}{8\left(M e^{\omega}+1\right)}
$$

последовательно получаем

$$
\begin{aligned}
& \left\|\mathscr{P}_{S}(\xi, v(\cdot))(t)-\mathscr{P}_{S}(\xi, v(\cdot))\left(t_{0}\right)\right\| \leqslant \| S(t-h)\left(S(h) \xi-S\left(t_{0}-t+h\right) \xi\right) \\
& \quad+\int_{0}^{t-h} S(t-h-\tau)\left(S(h)-S\left(t_{0}-t+h\right)\right) v(\tau) d \tau \| \\
& \quad+\int_{t-h}^{t}\left\|\left(S(t-\tau)-S\left(t_{0}-\tau\right)\right) v(\tau)\right\| d \tau+M e^{\omega} \int_{t}^{t_{0}}\|v(\tau)\| d \tau \\
& \leqslant\left\|S\left(t_{0}-t\right)-J\right\|\left\|S(h) \mathscr{P}_{S}(\xi, v(\cdot))(t-h)-x_{i}\right\|+\left\|S\left(t_{0}-t\right) x_{i}-x_{i}\right\| \\
& \quad+2 M e^{\omega} \int_{t-h}^{t}\|v(\tau)\| d \tau+M e^{\omega} \int_{t}^{t_{0}}\|v(\tau)\| d \tau \leqslant \varepsilon .
\end{aligned}
$$

Таким образом, множество $\mathscr{P}_{S}(K \times Q)$ равностепенно непрерывно в каждой точке $t_{0} \in(0,1]$ и утверждение леммы следует из теоремы Асколи [12, c. 304]. 
ЗАмЕчАниЕ 1 . В предположениях леммы 1 образ множества $K \times Q$ при отображении $\mathscr{P}_{S}$ будет также относительно компактньм в $L_{1}(T, X)$. В самом деле, пусть число $R>0$ таково, что

$$
\left\|\mathscr{P}_{S}(\xi, v(\cdot))(t)\right\| \leqslant R \quad \text { для всех } \xi \in K, \quad v(\cdot) \in Q, \quad t \in T,
$$

и последовательность $u_{n}(\cdot) \in \mathscr{P}_{S}(K \times Q)$ сходится к функции $u(\cdot)$ в пространстве $\left(C(T, X), \mathscr{T}_{C}\right)$. Тогда для любого $\varepsilon>0$, достаточно большого $m=1,2, \ldots$ и всех $n$ начиная с некоторого номера, будем иметь

$$
\int_{T}\left\|u_{n}(t)-u(t)\right\| d t \leqslant \frac{1}{m} R+\int_{0}^{1 / m}\|u(t)\| d t+p_{m}\left(u_{n}(\cdot)-u(\cdot)\right) \leqslant \varepsilon .
$$

3. Существование решений уравнения (1) в случае ограниченного множества. Пусть $\mathscr{U} \subset L_{1}(T, X), g: \mathscr{U} \rightarrow L_{1}(T, X)$, а $\boldsymbol{A}: D(\boldsymbol{A}) \rightarrow X$ - инфинитезимальный генератор $c_{0}$-полугруппы $S=\{S(t), t \geqslant 0\}$ линейных ограниченных операторов. Непрерьвная функция $u(\cdot) \in \mathscr{U}$ называется интегральным решением дифференциально-операторного уравнения (1), если для всех $t \in T$ имеет место равенство

$$
u(t)=S(t) u(0)+\int_{0}^{t} S(t-\tau) g(u)(\tau) d \tau .
$$

В случае, когда функция $u(\cdot)$ непрерьвно дифференцируема на $(0,1], u(t) \in D(\boldsymbol{A})$, и соотношение $(1)$ выполняется для всех $0<t \leqslant 1$, говорят, что $u(\cdot)$ является классическим решением (или просто решением) уравнения (1).

Для непустого замкнутого вьпуклого множества $K \subset X$ определим касательный конус к $K$ в точке $x \in K$ формулой

$$
T_{K}(x):=\overline{\bigcup_{\lambda>0} \frac{1}{\lambda}(K-x)}
$$

где черта сверху означает замькание в $X$ (см., например, [13, с. 219]). Очевидно, $v$ принадлежит $T_{K}(x)$ тогда и только тогда, когда

$$
\lim _{\lambda \rightarrow 0+} d_{K}(x+\lambda v)=0
$$

здесь $d_{K}(\cdot)$ - расстояние от точки до множества $K$ в пространстве $X$. Если $Q \subset L_{1}(T, X)$, то расстояние от точки до $Q$ в пространстве $L_{1}(T, X)$ мы обозначим символом $\mathscr{D}_{Q}(\cdot)$.

Всюду в дальнейшем, если не оговорено противное, банахово пространство $X$ будем считать сепарабельным.

Зафиксировав множество $K \subset X$ и суммируемую функцию $l(t) \geqslant 0$, положим

$$
\begin{gathered}
\mathscr{K}:=\left\{u(\cdot) \in L_{1}(T, X): u(t) \in K \text { для почти всех } t \in T\right\}, \\
Q:=\left\{v(\cdot) \in L_{1}(T, X):\|v(t)\| \leqslant 2(l(t)+1) \text { для почти всех } t \in T\right\}, \\
\mathscr{K}^{*}:=\overline{\mathscr{P}_{S}(K \times Q) \cap \mathscr{K}} .
\end{gathered}
$$

Здесь черта означает замыкание в $\left(C(T, X), \mathscr{T}_{c}\right)$. В случае ограниченного $K$ согласно лемме 1 и замечанию 1 оба пространства $\left(\mathscr{K}^{*}, \mathscr{T}_{c}\right)$ и $\left(\mathscr{K}^{*},\|\cdot\|_{1}\right)$ компактны.

Скажем, что $K$ инвариантно относительно полугруппы $S$, если $S(t) x \in K$ для всех $x \in K$ и $t \geqslant 0$. 
ТЕорема 1. Пусть непустое замкнутое выпуклое ограниченное множество $K$ инвариантно относительно компактной $c_{0}$-полугруппь $S$ с инфинитезимальным генератором $\boldsymbol{A}: D(\boldsymbol{A}) \rightarrow X$. Предположим, что непрерывное отображсене $g:\left(\mathscr{K}^{*},\|\cdot\|_{1}\right) \rightarrow L_{1}(T, X)$ для каждого $u(\cdot) \in \mathscr{K}^{*}$ удовлетворяет условиям

$$
\|g(u)(t)\| \leqslant l(t), \quad g(u)(t) \in T_{K}(u(t))
$$

почти всюду на T. Тогда для любой непрерывной функиии $\varphi:\left(\mathscr{K}^{*}, \mathscr{T}_{c}\right) \rightarrow K$ существует интегральное решение $u(\cdot) \in \mathscr{K}^{*}$ дифференциально-операторного уравнения (1) такое, что $u(0)=\varphi(u)$.

ДокАЗАТЕЛЬСтво. Для каждой функции $u(\cdot) \in \mathscr{K}^{*}$ и $\lambda>0$ по теореме 2.2 из [14]

$$
\mathscr{D}_{\mathscr{K}}(u+\lambda g(u))=\int_{T} d_{K}(u(t)+\lambda g(u)(t)) d t .
$$

Отсюда и из (7) по теореме Лебега об ограниченной сходимости следует, что

$$
\lim _{\lambda \rightarrow 0+} \frac{1}{\lambda} \mathscr{D}_{\mathscr{K}}(u+\lambda g(u))=0 \quad \text { для всех } u(\cdot) \in \mathscr{K}^{*} .
$$

Воспользовавшись теперь свойствами касательных конусов и компактностью множества $\mathscr{K}^{*}$ в $L_{1}(T, X)$, найдем [4, с. 211-212] последовательность $\lambda_{n} \rightarrow 0+$, для которой

$$
\frac{1}{\lambda_{n}} \mathscr{D}_{\mathscr{K}}\left(u+\lambda_{n} g(u)\right) \leqslant \frac{1}{n} \quad \text { для всех } u(\cdot) \in \mathscr{K}^{*}, \quad n=1,2, \ldots
$$

Как это делалось в леммах 5 и 6 из [1], определим непрерьвные функции $q_{n}: \mathscr{K} \rightarrow \mathscr{K}$, $n=1,2, \ldots$, удовлетворяюшие неравенствам

$$
\left\|u(t)+\lambda_{n} g(u)(t)-q_{n}(u)(t)\right\| \leqslant d_{K}\left(u(t)+\lambda_{n} g(u)(t)\right)+\frac{1}{n} \lambda_{n}
$$

для всех $u(\cdot) \in \mathscr{K}^{*}$ и почти всех $t \in T$,

$$
\left\|u(t)-q_{n}(u)(t)\right\| \leqslant 2 \lambda_{n}(l(t)+1)
$$

для всех $u(\cdot) \in \mathscr{K}$ и почти всех $t \in T$. При этом мы пользуемся результатами о непрерьвных селекторах, полученньми в [15].

Положим

$$
f(\xi, u)(t):=S(t) \xi+\int_{0}^{t} S(t-\tau) g(u)(\tau) d \tau
$$

для всех $\xi \in K, u(\cdot) \in \mathscr{K}^{*}, t \in T$ и

$$
\begin{gathered}
f_{n}(\xi, u)(t):=S(t) \xi+\frac{1}{\lambda_{n}} \int_{0}^{t} S(t-\tau)\left(q_{n}(u)(\tau)-u(\tau)\right) d \tau \\
\sigma_{n}(\xi, u)(t):=e^{-t / \lambda_{n}} S(t) \xi+\frac{1}{\lambda_{n}} \int_{0}^{t} e^{-(t-\tau) / \lambda_{n}} S(t-\tau) q_{n}(u)(\tau) d \tau
\end{gathered}
$$

для всех $\xi \in K, u(\cdot) \in \mathscr{K}, t \in T, n=1,2, \ldots$ 
Легко видеть, что отображения $f: K \times\left(\mathscr{K}^{*},\|\cdot\|_{1}\right) \rightarrow\left(C(T, X), \mathscr{T}_{c}\right), f_{n}: K \times \mathscr{K} \rightarrow$ $\left(C(T, X), \mathscr{T}_{c}\right)$ и $\sigma_{n}: K \times \mathscr{K} \rightarrow\left(C(T, X), \mathscr{T}_{c}\right)$ непрерьвны. Согласно $(9),(8)$ и теореме 2.2 из [14]

$$
\left\|f(\xi, u)(t)-f_{n}(\xi, u)(t)\right\| \leqslant \frac{M e^{\omega}}{\lambda_{n}} \int_{0}^{t} d_{K}\left(u(\tau)+\lambda_{n} g(u)(\tau)\right) d \tau+\frac{1}{n} M e^{\omega} \leqslant \frac{2}{n} M e^{\omega}
$$

$\xi \in K, u(\cdot) \in \mathscr{K}^{*}, t \in T, n=1,2, \ldots$. Непосредственными вычислениями проверяется справедливость равенства

$$
f_{n}(\xi, u)(t)-\sigma_{n}(\xi, u)(t)=\frac{1}{\lambda_{n}} \int_{0}^{t} S(t-\tau)\left(\sigma_{n}(\xi, u)(\tau)-u(\tau)\right) d \tau
$$

$\xi \in K, u(\cdot) \in \mathscr{K}, t \in T, n=1,2, \ldots$

Поскольку $K$ инвариантно относительно полугруппы $S$, то [1, лемма 1$] \sigma_{n}(\xi, u)(t) \in K$ для всех $\xi \in K, u(\cdot) \in \mathscr{K}, t \in T$. Пусть $R>0$ выбрано так, что $K \subset R \bar{B}$, и $Q_{n}:=\left\{v(\cdot) \in L_{1}(T, X):\|v(t)\| \leqslant R / \lambda_{n}+2(l(t)+1)\right.$ для почти всех $\left.t \in T\right\}$. Ясно, что $Q_{n}$ равномерно интегрируемо и оператор $\sigma_{n}$ переводит множество $K \times \mathscr{K}$ в $\mathscr{P}_{S_{n}}\left(K \times Q_{n}\right)$, где $S_{n}=\left\{S_{n}(t), t \geqslant 0\right\}, S_{n}(t):=e^{-t / \lambda_{n}} S(t)$, представляет собой компактную $c_{0}$-полугруппу линейных ограниченных операторов на $X$. Отсюда по лемме 1 следует, что множество $\sigma_{n}(K \times \mathscr{K})$ относительно компактно в пространствах $(C(T, X)$, $\left.\mathscr{T}_{c}\right)$ и $L_{1}(T, X)$.

Пусть $\varphi^{*}:\left(C(T, X), \mathscr{T}_{c}\right) \rightarrow K-$ непрерывная функция такая, что $\varphi^{*}(u)=\varphi(u)$ для всех $u(\cdot) \in \mathscr{K}^{*}$. Тогда для каждого $n=1,2, \ldots$ оператор $(\xi, u(\cdot)) \mapsto\left(\varphi^{*}\left(\sigma_{n}(\xi, u)\right)\right.$, $\left.\sigma_{n}(\xi, u)\right)$ переводит множество $K \times \mathscr{K}$ в его относительно компактное подмножество и является непрерывным. Применяя теорему Шаудера о неподвижной точке [4, с. 126], найдем пару $\left(\xi_{n}, u_{n}(\cdot)\right) \in K \times \mathscr{K}$, для которой $\xi_{n}=\varphi^{*}\left(\sigma_{n}\left(\xi_{n}, u_{n}\right)\right)$ и $u_{n}=\sigma_{n}\left(\xi_{n}, u_{n}\right)$. В частности, $u_{n}(0)=\xi_{n}$.

Из равенства (15) следует $f_{n}\left(\xi_{n}, u_{n}\right)=u_{n}, n \geqslant 1$. Так как согласно (12), (10) $u_{n}(\cdot) \in \mathscr{K}^{*}, n \geqslant 1$, то $\xi_{n}=\varphi\left(u_{n}\right)$ и из последовательности $\left\{u_{n}(\cdot)\right\}$ можно выделить подпоследовательность, сходящуюся как в $\left(C(T, X), \mathscr{T}_{c}\right)$, так и в $L_{1}(T, X)$. Не нарушая общности, считаем, что $u_{n}(\cdot) \rightarrow u(\cdot)$ при $n \rightarrow \infty$. Положим $\xi=\varphi(u)$. Тогда $\xi_{n} \rightarrow \xi$ и с учетом неравенства (14) в силу непрерьвности отображения $f: K \times\left(\mathscr{K}^{*},\|\cdot\|_{1}\right) \rightarrow$ $\left(C(T, X), \mathscr{T}_{c}\right)$ получим для каждого $m=1,2, \ldots$

$$
\begin{aligned}
p_{m}(u-f(\xi, u)) \leqslant & p_{m}\left(u-u_{n}\right)+p_{m}\left(f_{n}\left(\xi_{n}, u_{n}\right)-f\left(\xi_{n}, u_{n}\right)\right) \\
& +p_{m}\left(f\left(\xi_{n}, u_{n}\right)-f(\xi, u)\right) \rightarrow 0, \quad n \rightarrow \infty .
\end{aligned}
$$

Отсюда следует, что $u(t)=f(\xi, u)(t)$ для любого $t \in(0,1]$. Понятно, что равенство также справедливо при $t=0$. Таким образом, $u(0)=\xi=\varphi(u)$ и функция $u(\cdot) \in \mathscr{K}^{*}$ удовлетворяет соотношению (6). Теорема доказана.

При некоторых дополнительных предположениях поставленная задача имеет также классическое решение. Именно, используя доказанную теорему и предложение 4.3 из [4, с. 298], приходим к следующим следствиям.

СлЕДСТВИЕ 1. Пусть выполнены условия теоремы 1 , полугруппа $S=\{S(t)$, $t \geqslant 0\}$ является аналитической, а оператор g таков, что при каждом $и(\cdot) \in \mathscr{K}^{*}$ функиия $g(u)(\cdot)$ гёльдерова на T. Тогда уравнение (1) имеет классическое решение $u(\cdot) \in \mathscr{K}^{*}, \partial$ дя которого $u(0)=\varphi(u)$. 
СлЕДСТВИЕ 2. Пусть множество $K, c_{0}$-полугруппа $S$ линейных ограниченных операторов и отображение $g: \mathscr{K}^{*} \rightarrow L_{1}(T, X)$ такие же, как в теореме 1 . Тогда для любого из приведенных ниже краевых условий:

1) $u(0)=u(1)$

2) $u(0)=\sum_{i=1}^{n} \lambda_{i} u\left(\theta_{i}\right)$ при фиксированных $\theta_{i} \in(0,1] u \lambda_{i} \geqslant 0, i=1, \ldots, n$, $\sum_{i=1}^{n} \lambda_{i}=1$

3) $u(0)=\int_{0}^{1} u(t) d t$

найдется интегральное решение уравнения (1), удовлетворяющее этому условию. Кроме того, если выполнены предположения следствия 1, то каждое интегральное решение является классическим.

Для доказательства достаточно положить $\varphi(u)=u(1), \varphi(u)=\sum_{i=1}^{n} \lambda_{i} u\left(\theta_{i}\right)$ или $\varphi(u)=\int_{0}^{1} u(t) d t$ соответственно.

Функциональные краевые условия другого рода возникают в различных приложениях, в частности, к дифференциальным уравнениям с частньми производньги, что мы ниже проиллюстрируем на примере.

ЗАмЕчАниЕ 2. Если условие компактности наложить не на полугруппу $S$, а на множество $K$, то утверждения теоремы 1 и ее следствий останутся справедливыми, поскольку в этом случае, как видно из доказательства леммы 1 , множество $\mathscr{K}^{*}$ компактно в пространстве $C(T, X)$ непрерьвных функций с обычной нормой $\|\cdot\|_{C},\|x\|_{C}=\sup _{t \in T}\|x(t)\|$. Более того, в качестве $\varphi: \mathscr{K}^{*} \rightarrow K$ можно брать любое отображение, непрерьвное относительно введенной нормы на $\mathscr{K}^{*}$. При этом сепарабельность пространства $X$ можно не предполагать. В частности, когда $K \subset X$ компактно и $\boldsymbol{A} \equiv 0$, мы получаем существование решения $u(\cdot)$ уравнения $\dot{u}(t)=g(u)(t)$, удовлетворяющее условиям $(2),(3)$.

4. Существование решений: случай неограниченного множества. Если полугруппа $S$ является сжимающей, то полученньй в предыдущем разделе результат иногда удается обобщить на случай неограниченного множества $K$.

Теорема 2. Пусть $S$ - компактная сжимающая с $c_{0}$-полугруппа линейных ограниченных операторов с инфинитезимальным генератором $\boldsymbol{A}: D(\boldsymbol{A}) \rightarrow X, a$ $K$ - непустое замкнутое выпуклое, не обязательно ограниченное, подмножество $X$, инвариантное относительно данной полугруппь. Предполохим, что непрерывный оператор $g:\left(\mathscr{K}^{*},\|\cdot\|_{1}\right) \rightarrow L_{1}(T, X)$ удовлетворяет условиям (7) теоремы 1 , а непрерывное отображсене $\varphi:\left(\mathscr{K}^{*}, \mathscr{T}_{c}\right) \rightarrow K$ таково, что при некотором $0<\beta<1$

$$
\left\|\varphi(u)-\varphi\left(u^{\prime}\right)\right\| \leqslant \beta\left\|u-u^{\prime}\right\|_{C} \quad \text { для всех } \quad u(\cdot), u^{\prime}(\cdot) \in \mathscr{K}^{*} .
$$

Тогда уравнение (1) имеет интегральное решение $u(\cdot) \in \mathscr{K}^{*}$ такое, что $и(0)=\varphi(u)$.

ДоказАтЕльство. Согласно (16) оператор $\gamma: K \rightarrow K, \gamma(\xi)=\varphi(S(\cdot) \xi), \xi \in K$, является сжимающим и, следовательно, имеет единственную неподвижную точку. Обозначим ее через $\xi_{0}$.

Для фиксированного $L>0$ положим

$$
K_{L}:=\left\{\xi \in K:\left\|\xi-\xi_{0}\right\| \leqslant L\right\}, \quad \mathscr{K}_{0}^{*}:=\overline{\mathscr{P}_{S}\left(K_{L} \times Q\right) \cap \mathscr{K}}
$$


где $\mathscr{K}$ и $Q$ имеют тот же смысл, что и в п. 3 , а черта означает замыканиев $\left(C(T, X), \mathscr{T}_{c}\right)$. В силу леммы 1 множество $\mathscr{K}_{0}^{*}$ компактно в пространствах $\left(C(T, X), \mathscr{T}_{c}\right)$ и $L_{1}(T, X)$. Поэтому, повторяя доказательство теоремы 1 , мы можем, если необходимо, заменять $\mathscr{K}^{*}$ на $\mathscr{K}_{0}^{*}$. В частности, выберем последовательность $\lambda_{n} \rightarrow 0+$, для которой при всех $u(\cdot) \in \mathscr{K}_{0}^{*}$ справедливо неравенство (8), а также найдем непрерывные функции $q_{n}$ : $\mathscr{K} \rightarrow \mathscr{K}, n=1,2, \ldots$, удовлетворяющие $(9)$ для $u(\cdot) \in \mathscr{K}_{0}^{*}$ и $(10)$ для всех $u(\cdot) \in \mathscr{K}$.

Пусть непрерьвные отображения $f: K \times\left(\mathscr{K}^{*},\|\cdot\|_{1}\right) \rightarrow\left(C(T, X), \mathscr{T}_{c}\right), f_{n}: K \times \mathscr{K} \rightarrow$ $\left(C(T, X), \mathscr{T}_{c}\right)$ и $\sigma_{n}: K \times \mathscr{K} \rightarrow\left(C(T, X), \mathscr{T}_{c}\right), n \geqslant 1$, определены равенствами (11)-(13) соответственно. Положим

$$
\begin{aligned}
L & :=\frac{2 \beta}{1-\beta} \int_{T}(l(\tau)+1) d \tau, \quad r(t):=L+2 \int_{0}^{t}(l(\tau)+1) d \tau, \\
\mathscr{K}_{r} & :=\left\{x(\cdot) \in \mathscr{K}:\left\|x(t)-S(t) \xi_{0}\right\| \leqslant r(t) \text { для почти всех } t \in T\right\} .
\end{aligned}
$$

Тогда для $\xi \in K_{L}, u(\cdot) \in \mathscr{K}_{r}$ имеем

$$
\begin{aligned}
& \left\|\sigma_{n}(\xi, u)(t)-S(t) \xi_{0}\right\| \\
& \quad \leqslant e^{-t / \lambda_{n}}\left\|S(t) \xi-S(t) \xi_{0}\right\|+\lambda_{n}^{-1} \int_{0}^{t} e^{-(t-\tau) / \lambda_{n}}\left\|S(t-\tau) q_{n}(u)(\tau)-S(t) \xi_{0}\right\| d \tau \\
& \quad \leqslant L e^{-t / \lambda_{n}}+\lambda_{n}^{-1} \int_{0}^{t} e^{-(t-\tau) / \lambda_{n}}\left(2 \lambda_{n}(l(\tau)+1)+r(\tau)\right) d \tau=r(t),
\end{aligned}
$$

т.е. $\sigma_{n}(\xi, u) \in \mathscr{K}_{r} . \mathrm{C}$ другой стороны, для каждого $u(\cdot) \in \mathscr{K}_{r} \cap \mathscr{K}^{*}$ в силу (16)

$$
\left\|\varphi(u)-\xi_{0}\right\|=\left\|\varphi(u)-\varphi\left(S(\cdot) \xi_{0}\right)\right\| \leqslant \beta\left(L+2 \int_{T}(l(\tau)+1) d \tau\right)=L .
$$

Ясно, что $\varphi$ может быть продолжено до непрерьвного отображения $\varphi^{*}: \mathscr{K}_{r} \rightarrow K_{L}$. Следовательно, оператор

$$
\Phi_{n}:(\xi, u(\cdot)) \mapsto\left(\varphi^{*}\left(\sigma_{n}(\xi, u)\right), \sigma_{n}(\xi, u)\right)
$$

переводит множество $K_{L} \times \mathscr{K}_{r}$ в себя и является непрерьвным. Более того, множество $\Phi_{n}\left(K_{L} \times \mathscr{K}_{r}\right)$ относительно компактно в $X \times\left(C(T, X), \mathscr{T}_{c}\right)$. Находя по теореме Шаудера неподвижную точку $\left(\xi_{n}, u_{n}(\cdot)\right) \in K_{L} \times \mathscr{K}_{r}$ оператора (17), так же, как в теореме 1 , убеждаемся в том, что $u_{n}(\cdot) \in \mathscr{K}_{0}^{*}$ для всех $n \geqslant 1$, и предельная точка $u(\cdot) \in \mathscr{K}^{*}$ этой последовательности есть искомое интегральное решение уравнения $(1)$, удовлетворяющее функциональному краевому условию $u(0)=\varphi(u)$. Теорема доказана.

СЛЕДСТВИЕ 3. Пусть $c_{0}$-полугруппа $S$, множество $K \subset X$ и отображение $g: \mathscr{K}^{*} \rightarrow L_{1}(T, X)$ такие же, как в теореме 2 . Тогда для любых $\theta \in(0,1], \lambda \in[0,1)$ и $u_{0} \in K$ существует интегральное решение $u(\cdot) \in \mathscr{K}^{*}$ уравнения (1) такое, что $u(0)=\lambda u(\theta)+(1-\lambda) u_{0}$. 
5. Пример. Рассмотрим следующую краевую задачу:

$$
\begin{gathered}
\frac{\partial u}{\partial t}=\Delta u+F(t, x, u, \mathscr{R} u), \\
u(t, x)=0 \quad \text { для всех } x \in \partial \Omega, \quad t \in T, \quad u(0, x)=u(1, \psi(x)) \quad \text { для всех } x \in \bar{\Omega},
\end{gathered}
$$

где $\Omega$ - произвольная ограниченная область в $\mathbb{R}^{n}$ с гладкой границей $\partial \Omega$;

$$
\Delta=\sum_{i=1}^{n} \frac{\partial^{2}}{\partial x_{i}^{2}}
$$

- оператор Лапласа; $F: T \times \bar{\Omega} \times[a, b] \times[c, d] \rightarrow \mathbb{R},-\infty<a \leqslant 0 \leqslant b<+\infty, a<b$, $-\infty<c<d<+\infty ; \psi: \bar{\Omega} \rightarrow \bar{\Omega}$ и для каждой непрерывной функции $u, a \leqslant u(t, x) \leqslant b$, функция $\mathscr{R} u$ переменных $t, x$ непрерывна и принимает свои значения на отрезке $[c, d]$.

Пусть $X=C(\bar{\Omega})$ - пространство непрерьвных числовых функций на $\bar{\Omega}$ c sup-нормой. Определим

$$
\begin{aligned}
& D\left(\boldsymbol{A}_{C}\right):=\{u \in X: \Delta u \in X, u=0 \text { и } \Delta u=0 \text { на } \partial \Omega\} \\
& \left(\boldsymbol{A}_{C} u\right)(x):=\Delta u(x) \quad \text { для всех } \quad u \in D\left(\boldsymbol{A}_{C}\right), \quad x \in \bar{\Omega} .
\end{aligned}
$$

Согласно теореме 3.7 из [3, с. 217] $\boldsymbol{A}_{C}$ является инфинитезимальньп генератором аналитической $c_{0}$-полугрупшы $S_{C}=\left\{S_{C}(t), t \geqslant 0\right\}$.

Пусть $K:=\{u \in X: a \leqslant u(x) \leqslant b$ для всех $x \in \bar{\Omega}\}, \mathscr{W}:=\{w(\cdot) \in C(T, X): c \leqslant$ $w(t)(x) \leqslant d$ для всех $t \in T, x \in \bar{\Omega}\}$, а множества $\mathscr{K}$ и $\mathscr{K}^{*}-$ такие же, как в п. 3.

В дальнейшем предполагается, что

a) $t \mapsto F(t, x, u, w)$ измерима для всех $x \in \bar{\Omega},(u, w) \in[a, b] \times[c, d]$;

б) $(x, u, w) \mapsto F(t, x, u, w)$ непрерывна для почти всех $t \in T$;

в) для некоторой суммируемой функции $l(t) \geqslant 0$

$$
|F(t, x, u, w)| \leqslant l(t) \quad \text { для всех } x \in \bar{\Omega}, \quad(u, w) \in[a, b] \times[c, d]
$$

почти всюду на $T$;

г) оператор $\mathscr{R}:\left(\mathscr{K}^{*},\|\cdot\|_{1}\right) \rightarrow\left(\mathscr{W},\|\cdot\|_{1}\right)$ непрерывен.

Если для каждого $u(\cdot) \in \mathscr{K}^{*}, t \in T$ через $g(u)(t)$ мы обозначим отображение $x \mapsto F(t$, $x, u(t)(x), \mathscr{R} u(t)(x))$, то оператор $g$ переводит множество $\mathscr{K}^{*}$ в $L_{1}(T, X)$. Легко также проверить, что он непрерьвен относительно нормы $\|\cdot\|_{1}$ на $\mathscr{K}^{*}$.

Как это принято, непрерывную функцию $u$ переменных $t, x$ назовем $X$-решением ( интегральным $X$-решением) задачи (18), если $t \mapsto u(t, \cdot)$ является классическим (соответственно интегральным) решением эволюционного уравнения $\dot{u}(t)=\boldsymbol{A}_{C} u(t)+g(u)(t)$ с функциональным краевым условием $u(0)=\varphi(u)$, где $\varphi: \mathscr{K}^{*} \rightarrow K$,

$$
\varphi(u)(x):=u(1)(\psi(x)) \quad \text { для всех } u(\cdot) \in \mathscr{K}^{*}, \quad x \in \bar{\Omega} .
$$

При этом мы естественным образом отождествляем элементы пространств $C(T \times \bar{\Omega}, \mathbb{R})$ и $C(T, X)$. 
Теорема 3. Пусть наряду с условиями а)-г) справедливы неравенства

$$
F(t, x, a, w) \geqslant 0, \quad F(t, x, b, w) \leqslant 0
$$

для почти всех $t \in T$ и всех $x \in \bar{\Omega}, w \in[c, d]$. Тогда для любого непрерывного отображсния $\psi: \bar{\Omega} \rightarrow \bar{\Omega}$ задача (18) имеет интегральное $X$-решение $u, a \leqslant u(t, x) \leqslant b$ для всех $t \in T, x \in \bar{\Omega}$.

ДокАЗАТЕЛЬСтво. Отметим, во-первых, что в силу теорем 1.2 и 3.1 из [3, с. 208, c. 212] оператор $\left(\boldsymbol{A}_{C}-\lambda J\right)^{-1}$ является компактньм, как только он ограничен. Отсюда и из следствия 5.1 из [4, с. 303] вытекает компактность полугрупшы $S_{C}$. Для любого $z \in K$ согласно дифференцируемости полугруппы функция $u(t, x)=\left(S_{C}(t) z\right)(x)$ непрерьвна на множестве $\{(t, x): t \geqslant 0, x \in \bar{\Omega}\}$ и дважды непрерьвно дифференцируема на его внутренности. Следовательно, по принципу максимума (см., например, [16, с. 511]) для всех $t \in T, x \in \bar{\Omega}$ имеем

$$
a \leqslant \min \left\{0, \min _{x \in \bar{\Omega}} z(x)\right\} \leqslant u(t, x) \leqslant \max \left\{0, \max _{x \in \bar{\Omega}} z(x)\right\} \leqslant b .
$$

Тем самым, множество $K$ инвариантно относительно данной полугрупш.

Зафиксируем $u(\cdot) \in \mathscr{K}^{*}$. Для того чтобы доказать включение $g(u)(t) \in T_{K}(u(t))$, возьмем $t \in T$, при котором имеют место б) и (20). Воспользовавшись этими условиями, а также непрерьвностью функций $u(t), \mathscr{R} u(t)$ и компактностью множества $\bar{\Omega}$, для любого $\varepsilon>0$ найдем $\delta>0$ такое, что

$$
P_{\lambda}(x):=[a, b] \cap(u(t)(x)+\lambda F(t, x, u(t)(x), \mathscr{R} u(t)(x))+(-\varepsilon \lambda, \varepsilon \lambda)) \neq \varnothing
$$

для всех $x \in \bar{\Omega}_{\text {и }} 0<\lambda \leqslant \delta$. По теореме Майкла [13, с. 82] многозначное отображение $\overline{P_{\lambda}}: \bar{\Omega} \rightarrow \mathbb{R}, \overline{P_{\lambda}}(x)=\overline{P_{\lambda}(x)}$, имеет непрерьвньй селектор $z_{\lambda}, a \leqslant z_{\lambda}(x) \leqslant b$, с помощью которого получаем

$$
\frac{1}{\lambda} d_{K}(u(t)+\lambda g(u)(t)) \leqslant \frac{1}{\lambda} \sup _{x \in \bar{\Omega}}\left|u(t)(x)+\lambda F(t, x, u(t)(x), \mathscr{R} u(t)(x))-z_{\lambda}(x)\right| \leqslant \varepsilon .
$$

Очевидно, $\|g(u)(t)\| \leqslant l(t)$ для почти всех $t \in T$ и функция $\varphi: \mathscr{K}^{*} \rightarrow K$, определенная равенством (19), непрерьвна относительно топологии $\mathscr{T}_{c}$ на $\mathscr{K}^{*}$. Для завершения доказательства теперь достаточно воспользоваться теоремой 1.

СлЕдСТВИЕ 4. Если дополнительно к условиям теоремы 3 функиия $F$ удовлетворяет условию Гёльдера по $t, u, w$, т.е. существуют числа $N>0 u 0<\alpha \leqslant 1$ такие, что

$$
\left|F(t, x, u, w)-F\left(t^{\prime}, x, u^{\prime}, w^{\prime}\right)\right| \leqslant N\left(\left|t-t^{\prime}\right|^{\alpha}+\left|u-u^{\prime}\right|^{\alpha}+\left|w-w^{\prime}\right|^{\alpha}\right)
$$

для всех $x \in \bar{\Omega}, t, t^{\prime} \in T,(u, w),\left(u^{\prime}, w^{\prime}\right) \in[a, b] \times[c, d]$, а функиия $\mathscr{R} u(\cdot)$ гёльдерова на $T$ для любой $u(\cdot) \in \mathscr{K}^{*}$, то краевая задача (18) имеет X-решение и, принимающее значения в отрезке $[a, b]$.

Данное утверждение непосредственно вытекает из предыдущей теоремы и предложений $4.3,4.4$ из [4, с. 298-300].

В качестве примера оператора $\mathscr{R}$ со свойствами, указанными в теореме 3 и следствии 4, отметим линейный интегральньй оператор

$$
\mathscr{R} u(t)(x)=\int_{T} G(t, \tau, x) u(\tau)(x) d \tau,
$$

ядро которого ограничено и является гёльдеровым по $t$ равномерно по $\tau \in T$ и $x \in \bar{\Omega}$. 


\section{СПИСОК ЦИТИРОВАННОЙ ЛИТЕРАТУРЫ}

[1] Гончаров В.В. О существовании решений одного класса дифференциальных включений на компактном множестве // Сиб. матем. ж. 1990. Т. 31. № 5. С. 24-30.

[2] Гончаров В.В.Теоремы существования решений дифференциальных включений с полунепрерывной снизу правой частью на локально компактном множестве // Дифференц. уравнения. 1991. Т. 27. № 12 . С. 2058-2065.

[3] Pazy A. Semigroups of Linear Operators and Applications to Partial Differential Equations. New York: Springer, 1983.

[4] Martin R. H. Nonlinear Operators and Differential Equations in Banach Spaces. New York: Wiley, 1976.

[5] Toshiyuki I. Global existence of mild solutions to semilinear differential equations in Banach spaces // Hiroshima Math. J. 1986. V. 16. P. 499-530.

[6] Deimling K. Periodic solutions of differential equations in Banach spaces // Manuscripta Math. 1978. V. 24. P. 31-44.

[7] Prüss J. Periodic solutions of semilinear evolution equations // Nonlinear Anal. 1979. V. 3. № 5. P. 601-612.

[8] Брыкалов С. А. Существование и неединственность решений некоторых нелинейных краевых задач // Докл. РАН. 1991. Т. 316. №1. С. 18-21.

[9] Брыкалов С. А. Разрешимость нелинейной краевой задачи в фиксированном множестве функций // Дифференц. уравнения. 1991. Т. 27. №12. С. 2027-2033.

[10] Brykalov S. A. A second-order nonlinear problem with two-point and integral boundary conditions // Proc. Georgian Acad. Sci. Math. 1993. V. 1. № 3. P. 273-279.

[11] Deimling K. Ordinary Differential Equations in Banach Spaces. Berlin: Springer, 1977.

[12] Келли Дж. Л. Общая топология. М.: Наука, 1981.

[13] Aubin J.-P., Cellina A. Differential Inclusions. Berlin: Springer, 1984.

[14] Hiai F., Umegaki H. Integrals, conditional expectations, and martingales of multivalued functions // J. Multivariate Anal. 1977. V. 7. P. 149-182.

[15] Bressan A., Colombo G. Extensions and selections of maps with decomposable values // Studia Math. 1988. V. 90. №1. P. 69-86.

[16] Владимиров В. С. Уравнения математической физики. М.: Наука, 1976. 